Ann. Zootech., I977, 26 (I), 93-97.

\title{
Utilisation du tourteau de colza dans l'alimentation du lapin en croissance Influence du dépelliculage
}

\author{
F. LEBAS et M. COLIN \\ avec la collaboration technique de G. SARDI \\ Laboratoire de Recherches sur l'Élevage du Lapin, \\ Centre national de Recherches zootechniques I.N.R.A., \\ 78350 Jouy-en-Josas (France)
}

\begin{abstract}
Résumé
Quarante-cinq lapins âgés de 35 jours ont reçu à volonté durant 6 semaines un des aliments expérimentaux dans lesquels $3 \mathrm{I}$ p. Ioo des protéines étaient apportées respectivement par du tourteau de colza toasté, du tourteau de colza dépelliculé puis toasté ou du tourteau de tournesol, les 3 régimes ayant une teneur en protéines de I6 p. Ioo. Les performances de croissance, de consommation et d'efficacité alimentaires sont identiques pour les 3 tourteaux. De même les poids des thyroïdes et du foie ne sont pas affectés par la consommation du tourteau de colza dépelliculé ou non.
\end{abstract}

\section{Introduction}

Des travaux antérieurs ont montré la possibilité d'emploi du tourteau de colza (Brassica oleracea) dans l'alimentation du lapin en croissance (Colin et LEBAS, I976). Par ailleurs l'élimination des pellicules des graines de colza facilite le raffinage des huiles et en outre réduit de moitié la teneur en cellulose des tourteaux (CHANeT, I972) ce qui représente un intérêt certain pour des monogastriques comme le porc ou le poulet. Cependant, les thioglucosides, responsables de l'effet goitrigène et de la réduction de croissance chez certaines espèces (VANETTEN, I969) étant concentrés principalement dans les cotylédons (EARLE et al., r966) on pourrait craindre que le dépelliculage n'entraîne un effet nocif chez le lapin par concentration du tourteau en ces substances. En effet les enveloppes représentent environ 30 p. Ioo du poids total du tourteau deshuilé (CHANET, I972). Il nous a donc semblé 
utile d'entreprendre l'étude de la valeur alimentaire du tourteau de colza dépelliculé en comparaison d'une part avec un tourteau de colza normal et d'autre part avec un tourteau de tournesol.

\section{TABLEAU I}

Composition des régimes (en p. Ioo)

Composition of the experimental diets ( $p$. Ioo)

\begin{tabular}{|c|c|c|c|}
\hline $\begin{array}{l}\text { Tourteaux } \\
\text { (Oil meal) }\end{array}$ & $\begin{array}{c}\text { Colza normal } \\
\text { (Standard Rapeseed) }\end{array}$ & $\begin{array}{l}\text { Colza dépelliculé } \\
\text { (Dehulled Rapeseed) }\end{array}$ & $\begin{array}{c}\text { Tournesol } \\
\text { (Sunflower) }\end{array}$ \\
\hline & & $T 5$ & \\
\hline Blé (Wheat) & 22,38 & 21,88 & 23,82 \\
\hline $\begin{array}{l}\text { Son (Wheat bran) } \\
\text { T. de colza (1) (Rapeseed oil }\end{array}$ & Io & Io & Io \\
\hline T. de colza dépelliculé ( $\left.{ }^{2}\right)(D e-$ & $\mathrm{r} 5,5$ & 一 & - \\
\hline $\begin{array}{l}\text { hulled Rapeseed oil meal) } \\
\mathrm{T} . \text { de tournesol ( }{ }^{(3)} \text { (Sunflower }\end{array}$ & - & I3 & - \\
\hline $\begin{array}{l}\text { oil meal) } \\
\text { Farine de luzerne déshydratée }\end{array}$ & $\longrightarrow$ & - & $\mathbf{I} 2$ \\
\hline (dehydrated lucerne) . . . . & 33 & 34 & 33 \\
\hline $\begin{array}{l}\text { Paille d'orge (Barley straw) }) \\
\mathrm{L} \text { arginine } \mathrm{HCl}(L \text { arginine }\end{array}$ & 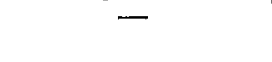 & 2 & $\mathbf{I}$ \\
\hline$H(l)$. . . . . . . . & 0,12 & 0,12 & - \\
\hline $\begin{array}{l}\mathrm{L} \text { lysine } \mathrm{HCl}(L \text { lysine } H C l) \\
\mathrm{DL} \text { méthionine }(D L \text { methio- }\end{array}$ & - & - & $0, \mathbf{I} 3$ \\
\hline $\begin{array}{l}\text { nine) } \\
\text { Complément minéral (4) vita- } \\
\text { minique (5) (Minerals and }\end{array}$ & - & - & 0,05 \\
\hline vitamins) . . . . . . . . . & 4 & 4 & 4 \\
\hline
\end{tabular}

(1) Variété Primor, toasté, 32,2 p. roo de matières azotées - - I3, I p. roo de cellulose brute. (Variety: Primor, toasted - 32,2 p. roo crude protein - I3,I p. Ioo crude fibre.)

(2) Variété Primor, dépelliculé avant extraction et toastage 38,4 p. Ioo de matières azotées $-8,7$ p. roo de cellulose brute. (Variety :Primor, seeds first dehulled and then oil extracted and toasted $-38,4 p .100$ crude protein and 8,7 p. roo crude fibre.)

(3) 4I,4 p. xoo de matieres azotées - I4,4 p. Ioo de cellulose brute. (4I,4 p. roo crude protein - I4,4 p. Ioo crude fibre.)

(4) Mineraux en p. roo du C.M. (Minerals $p$. too of the total mineral added.)

\begin{tabular}{|c|c|}
\hline Phosphate bicalcique (Dicalcium Phosphate) & 34,8 \\
\hline Chlorure de sodium (Sodium Chloride) & 23 \\
\hline Phosphate disodique (Disodium Phosphate) & 23 \\
\hline $\begin{array}{l}\text { Carbonate de magnésium (Magnesium Carbo- } \\
\text { nate) }\end{array}$ & I 8 \\
\hline Iodure de potassium $(I K)$ & 0,006 \\
\hline Sulfate de cuivre $\left(5 \mathrm{H}_{2} \mathrm{O}\right) \quad\left(\mathrm{SO}_{4} \mathrm{Cu},{ }_{5} \mathrm{H}_{2} \mathrm{O}\right)$ & 0,05 \\
\hline Sulfate de zinc $\left(\mathrm{I} \mathrm{H}_{2} \mathrm{O}\right)\left(\mathrm{SO}_{4} \mathrm{Zn}, \mathrm{I} \mathrm{H}_{2} \mathrm{O}\right)$ & 0,15 \\
\hline Sulfate de cobalt $\left(7 \mathrm{H}_{2} \mathrm{O}\right)\left(\mathrm{SO}_{4} \mathrm{Co}\right.$, & 0,09 \\
\hline Sulfate de manganèse $\left(\mathrm{H}_{2} \mathrm{O}\right)\left(\mathrm{SO}_{4} M n,{ }_{I} \mathrm{H}_{2} \mathrm{O}\right)$ & 0,09 \\
\hline Sulfate de fer $\left(7 \mathrm{H}_{2} \mathrm{O}\right)\left(\mathrm{SO}_{4} \mathrm{Fe},{ }_{7} \mathrm{H}_{2} \mathrm{O}\right)$ & \\
\hline
\end{tabular}

(5) Vitamines - (Vitamins) : CoLrs, 1975. 


\section{Matériel et méthodes}

Les quarante-cinq lapins des 2 sexes, de race "Californien " âgés de 35 jours au début de l'expérience ont été répartis entre les 3 traitements expérimentaux. Ils ont été logés dans des cages individuelles entièrement métalliques munies d'un abreuvoir à surface d'eau libre. La croissance des animaux a été mesurée par des pesées hebdomadaires et la consommation alimentaire contrôlée par pesée des quantités distribuées et refusées, 2 fois par semaine. Six semaines après le début de l'expérience, les animaux ont été sacrifiés et les poids des 2 thyroïdes (recherche de l'effet goitrigène) et du foie (compte tenu de son rôle dans une détoxication éventuelle) ont été mesurés.

Les 3 aliments relatifs aux 3 traitements expérimentaux ont une composition du type habituellement utilisé pour l'alimentation des lapins en croissance (tabl. I). Les 2 tourteaux de colza proviennent du même lot de graines (variété Primor) et ont été toastés dans les 2 cas. Le dépelliculage de l'un des lots, avant extraction de l'huile, a été réalisé selon les modalités décrites par Chanet (r972). Les 3 ali-

\section{TABLEAU 2}

Performances de croissance des animaux

(Growth performances of rabbits)

- I5 lapins par traitement

- durée de l'expérience : 6 semaines

- I5 animals for each treatment

- duration of experiment : 6 weeks

\begin{tabular}{|c|c|c|c|c|}
\hline $\begin{array}{c}\text { Régimes } \\
\text { (diets) }\end{array}$ & $\begin{array}{l}\text { Colza normal } \\
\text { (Standard } \\
\text { Rapeseed) }\end{array}$ & $\begin{array}{c}\text { Colza } \\
\text { dépelliculé } \\
\text { (Dehulled } \\
\text { Rapeseed) }\end{array}$ & $\begin{array}{c}\text { Tournesol } \\
\text { (Sunflower) }\end{array}$ & $\begin{array}{c}\text { Signification } \\
\text { statistique } \\
\text { (F calcule) } \\
\text { (Statistical } \\
\text { significance } \\
\text { calculated F) }\end{array}$ \\
\hline $\begin{array}{l}\text { Poids en début d'expé- } \\
\text { rience (g) (Weight at } \\
\text { the beginning) . . . }\end{array}$ & $918 \quad \pm 22$ & $919 \pm 22$ & $918 \pm 24$ & - \\
\hline $\begin{array}{l}\text { Gain de poids quotidien } \\
(\mathrm{g} / \mathrm{j})(\text { Daily weight- } \\
\text { gain }-g / d) \quad . .\end{array}$ & $35,5 \pm 1,2$ & $35,4 \pm 1, I$ & $36,5 \pm \quad 1,2$ & $<$ I NS (a) \\
\hline $\begin{array}{l}\text { Consommation }(\mathrm{g} / \mathrm{j}) \\
(\text { Daily food intake- } \\
g(d) \quad \cdot \cdot \cdot \cdot \cdot \cdot \cdot\end{array}$ & $127 \pm 3$ & $128 \pm 3$ & I32 $\pm \mathbf{3}$ & $<$ I NS \\
\hline $\begin{array}{l}\text { Indice de consomma- } \\
\text { tion }(\text { Feed conversion } \\
\text { ratio }) . . . .\end{array}$ & $3,6 \mathrm{r} \pm 0,08$ & $3,64 \pm 0,08$ & $3,63 \pm 0,06$ & $<$ I NS \\
\hline
\end{tabular}

(1) \pm fart type de la moyenne ( \pm standard deviation from mean.)

(2) Non significatif. (Non significant.) 
TABLEAU 3

Poids des glandes thyroides et du foie

Weight of the 2 thyroid glands and liver

\begin{tabular}{|c|c|c|c|c|}
\hline $\begin{array}{r}\text { Régimes } \\
\text { (diets) }\end{array}$ & $\begin{array}{l}\text { Colza INormal } \\
\text { (Standavd } \\
\text { Rapeseed) }\end{array}$ & $\begin{array}{c}\text { Colza } \\
\text { dépelliculé } \\
\text { (Dehulled } \\
\text { Rapeseed })\end{array}$ & $\begin{array}{l}\text { Tournesol } \\
\text { (Sunflower) }\end{array}$ & $\begin{array}{c}\text { Signification } \\
\text { statistique } \\
\text { (F calculé) } \\
\text { (Statistical } \\
\text { Significance } \\
\text { - calculated F) }\end{array}$ \\
\hline $\begin{array}{l}\text { Poids des animaux à. } \\
\text { l'abattage ( }{ }^{(1)}(\mathrm{g}) \\
\text { (Live weight at } \\
\text { slaughter }-g) .\end{array}$ & $2404 \pm 64\left({ }^{2}\right)$ & $2408 \pm 50$ & $244^{8} \pm 66$ & - \\
\hline $\begin{array}{lr}\text { Poids des } 2 & \text { glandes } \\
\text { thyroïdes } & (\mathrm{g}) \\
\text { (Weight of } 2 & \text { thy- } \\
\text { roid glands } & - \\
\end{array}$ & $0,22 \pm 0,01$ & $0,25 \pm 0,02$ & $0,23 \pm 0,02$ & $\mathrm{I}, \mathrm{O}$ NS $\left({ }^{3}\right)$ \\
\hline $\begin{array}{l}\text { Poids du foie (g) } \\
\quad \text { (Weight of liver-g) }\end{array}$ & $93,7 \pm 4,8$ & $96, \mathrm{I} \pm 4,2$ & $86, \mathrm{I} \pm 5,7$ & $\mathrm{I}, \mathrm{I} \mathrm{NS}$ \\
\hline
\end{tabular}

(1) Animaux âgés de II semaines. (At the age of II weeks.)

${ }^{(2)}$ Écart-type de la moyenne - (Standard deviation from mean.)

(3) Non significatif. (Non significant).

ments iso-protéiques (I6 p. I00) et iso-cellulosiques ( 13 p. Ioo) ont été formulés de sorte que le tourteau étudié apporte $3 \mathrm{I}$ p. Ioo des protéines totales. Des supplémentations en acides aminés purifiés ont été effectuées pour rendre les régimes isolysine $(0,70 \mathrm{p}$. IOo), iso-arginine $(0,92 \mathrm{p}$. IOo) et iso-acides aminés soufrés $(0,60 \mathrm{p}$. IOO).

\section{Résultats}

Les performances de croissance, de consommation et d'efficacité alimentaires sont identiques pour les 3 tourteaux étudiés (tabl. 2). Parallèlement, les thyroïdes et le foie ont des poids comparables pour les animaux des 3 catégories (tabl. 3 ). Même pour ce dernier organe, la très faible valeur du F de FisHer permet d'affirmer que la différence apparemment observée sur les moyennes est d'origine aléatoire.

\section{Discussion}

Les résultats obtenus confirment les observations antérieures montrant une bonne utilisation du tourteau de colza simplement toastés, par le lapin en croissance (Colin et LEBAS, I976). En outre, on ne relève, avec ce produit, pas d'effet goitrigène apparent, bien que le taux d'incorporation soit de I5 p. Ioo. Nous obtenons donc des résultats comparables à ceux enregistrés chez le chien par BRown, 
HoAG et BRACKEN (I974). En effet nous ne retrouvons pas les effets défavorables signalés pour le rat, le porc, la poule pondeuse (VANETTEN, I969), le pintadeau et le poulet (Blum, Guillaume et Leclerco, I973) ou les ovins (Theriez, Grenet et Molenat, I97I).

Par ailleurs, le dépelliculage n'a entraîné aucune modification de la valeur alimentaire du tourteau de colza, quand on considère des régimes iso-azotés et iso-cellulosiques. L'hypothèse, initiale du risque d'apparition d'effets nocifs dus à la concentration en isothiocyanate dans le tourteau dépelliculé ne s'avère donc pas fondée.

En conclusion, il semble donc possible d'utiliser jusqu'à I5 p. Ioo au moins, le tourteau de colza toasté, dépelliculé ou non, dans l'aliment des lapins en croissance; toutefois cela ne préjuge en rien des effets toxiques pouvant se manifester éventuellement chez les lapins reproducteurs.

Reçu pour publication en décembre 1976.

\section{Remerciements}

Les auteurs tiennent à remercier le C.E.T.I.O.M. pour la fourniture des différents tourteaux.

\section{Summary}

\section{Utilization of rapeseed oil-meal in growing rabbit feeding. Effect of dehulling}

Fourty five 35 days old rabbits received ad libitum for 6 weeks one of the three experimental diets in which $31 \%$ of the proteins were supplied by either toasted rapeseed oil meal, or dehulled and toasted rapeseed oil meal or sunflower oil meal, the protein content being $16 \%$ in all diets. Growth performances, feed intake and feed efficiency were the same for the three oil meals. Likewise, weights of thyroïd glands and liver were not affected by the intake of hulled or dehulled rapeseed oil meal.

\section{Références bibliographiques}

Blum J. C., Guillaume J., LeclercQ B., I973. Influence du tourteau de colza sur la croissance du pintadeau. Comparaison avec le poulet. Journées Rech. Avicole et Cunicole I79-I82, I.T.A.V.I. éd. Paris.

Brown R. G., Hoag G. N., Bracken E., i974. Rapeseed meal for dogs. Proc. Nut. Conf. Feed Mf. Univ. Guelph. April 98-roo.

Chanet M., i 972 . Depelliculage des graines de colza. i 8 p. CETIOM éd. Paris.

Colin M., I975. Effets sur la croissance du lapin de la supplémentation en L lysine et en DL méthionine de régimes végétaux simplifiés. Ann. Zootech., 24, 465-474.

Colin M., Lebas F., i976. Emploi du tourteau de colza, de la féverole et du pois dans les aliments pour le lapin en croissance. ${ }_{\text {er }}^{\mathrm{r}}$ congrès int. Cunicole, Dijon 1976 - communication $n^{0} 24$.

Earle F. R., Peters J. E., Wolff I. A., White G. A., Ig66. Compositional differences among crambe samples and between seed components. J. am. Oil Chemist's Soc. 43, 330-333.

Theriez M., Grenet N., Molenat G., i97I. Le tourteau de colza dans l'alimentation animale. IV. -- étude comparée de l'appétibilité et de la valeur alimentaire des tourteaux de colza et de lin pour l'agneau à l'entraissement et la brebis gestante; effets sur la glande thyroïde. Ann. Zootech., 20, 45I-463.

Vanetten G. G., in Lrener I. E., 1969. Toxic constituents of plant foodstuffs. IO3-I4I. Academic Press N.Y. 